\title{
Integral Field Spectrography of Active Galaxies with TIGER
}

\author{
P. Ferruit, E. Pécontal, G. Adam, and R. Bacon \\ CRAL Observatoire de Lyon, UMR CNRS 5574, F-69561 \\ Saint-Genis-Laval Cedex, France
}

\begin{abstract}
The TIGER integral field spectrograph has been used to study nearby and distant active galaxies. We first present new Tiger observations of the Seyfert galaxy NGC 1275 . In this very complex object, TIGER allows us to disentangle the contributions of the numerous components. We present the first very promising results on NGC 5252 obtained with the 3-D data-cube deconvolution techniques developed by our team in Lyon.
\end{abstract}

\section{TIGER Observations of Active Galaxies}

Talks and posters throughout this conference bear witness to the growing success of bidimensional spectrography in astronomy. The TIGER instrument (Bacon et al. 1995) developed in Lyon for the CFH telescope belongs to the first generation of these spectrographs. It has been successfully used to observe active galaxies and nearby Seyferts (Pécontal \& Ferruit 1994; Ferruit \& Pécontal 1994; Pécontal et al. 1995), and more distant objects such as radio galaxies (Adam et al. 1996; Rocca-Volmerange et al. 1994) and quasars (Durret et al. 1994; Petitjean et al. 1996). In the following, we first present new TIGER observations of the Seyfert galaxy NGC 1275 . This example of an extremely complex object illustrates well the power of TIGER. We also show first results obtained on NGC 5252 using new promising 3-D data cube deconvolution techniques developed in Lyon.

\section{NGC 1275: The Challenge of Complexity}

NGC 1275 is distinguished by its complexity (see Ferruit \& Pécontal 1994 and references therein). This $\mathrm{cD}$ galaxy lies in the center of the Perseus cluster and is one of the most powerful X-ray-selected cooling-flow candidates. It hosts a Seyfert 1 nucleus, a strong radio source with a radio jet, and a system of star clusters. But one of the most interesting feature of this galaxy is the line-ofsight superposition of a very extended system of line-emitting filaments, the low-velocity (LV) component, with $\mathrm{H} \alpha$ clumps that are likely to belong to a $+3000 \mathrm{~km} \mathrm{~s}^{-1}$ infalling galaxy, which produces the so-called high-velocity (HV) system.

New TIGER $0^{\prime \prime} .8$ spatial-resolution observations of this object at low $(18 \AA)$ and medium $(3.6 \AA)$ spectral resolution were obtained in 1994 November at 

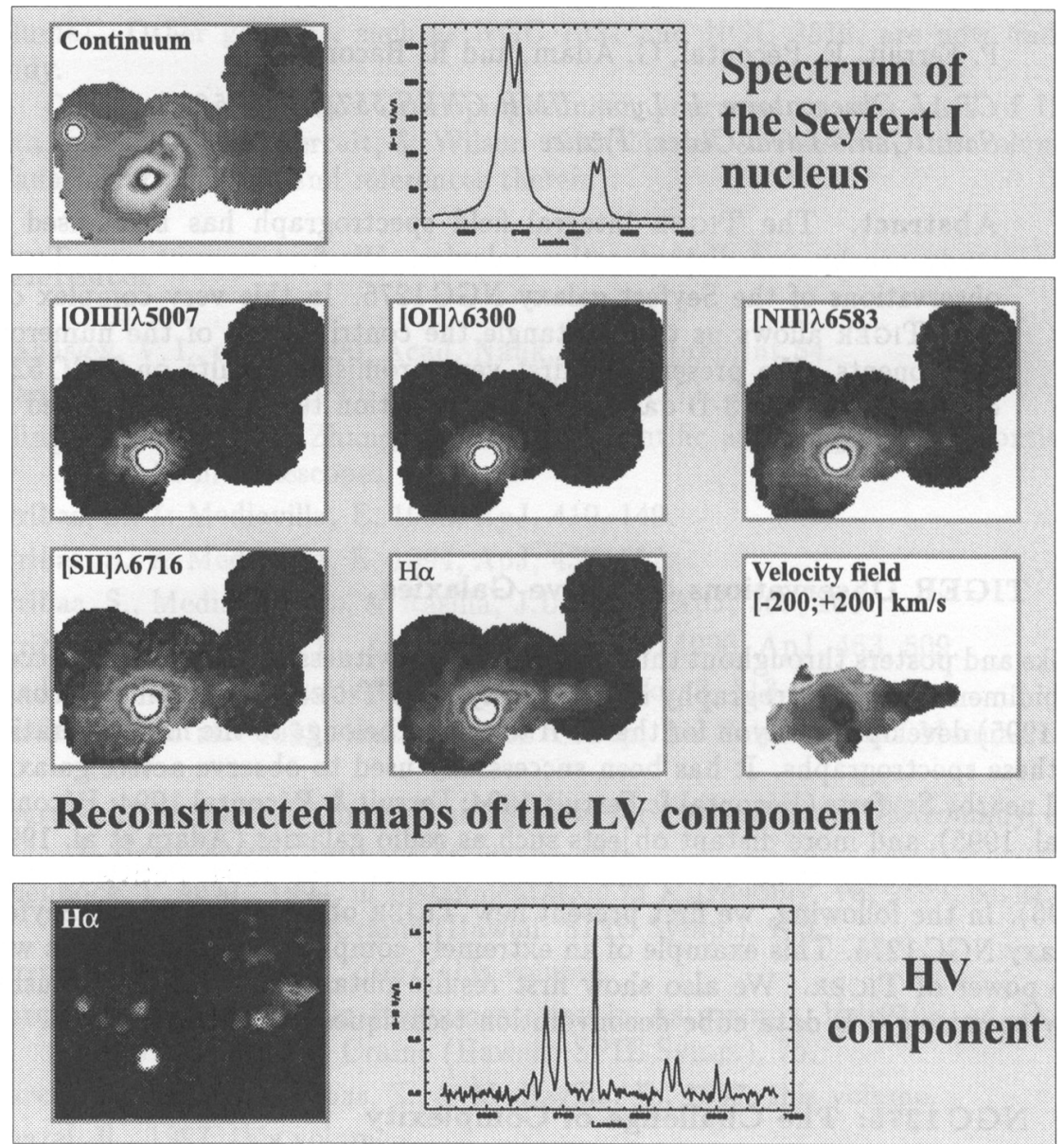

Figure 1. Top: Continuum map with a spectrum of the nucleus. Middle: Maps of the LV component in various lines and the associated velocity field. Bottom: $\mathrm{H} \alpha$ map of the $\mathrm{HV}$ component (the bright peak at the location of the nucleus is an artifact due to contamination by the broad nuclear $\mathrm{H} \alpha$ ). Typical spectrum displaying the [N $\mathrm{NI}], \mathrm{H} \alpha$ and [S II] lines of the LV and HV systems. Note the very high $\mathrm{H} \alpha /[\mathrm{N}$ II] flux ratio of the HV system. 


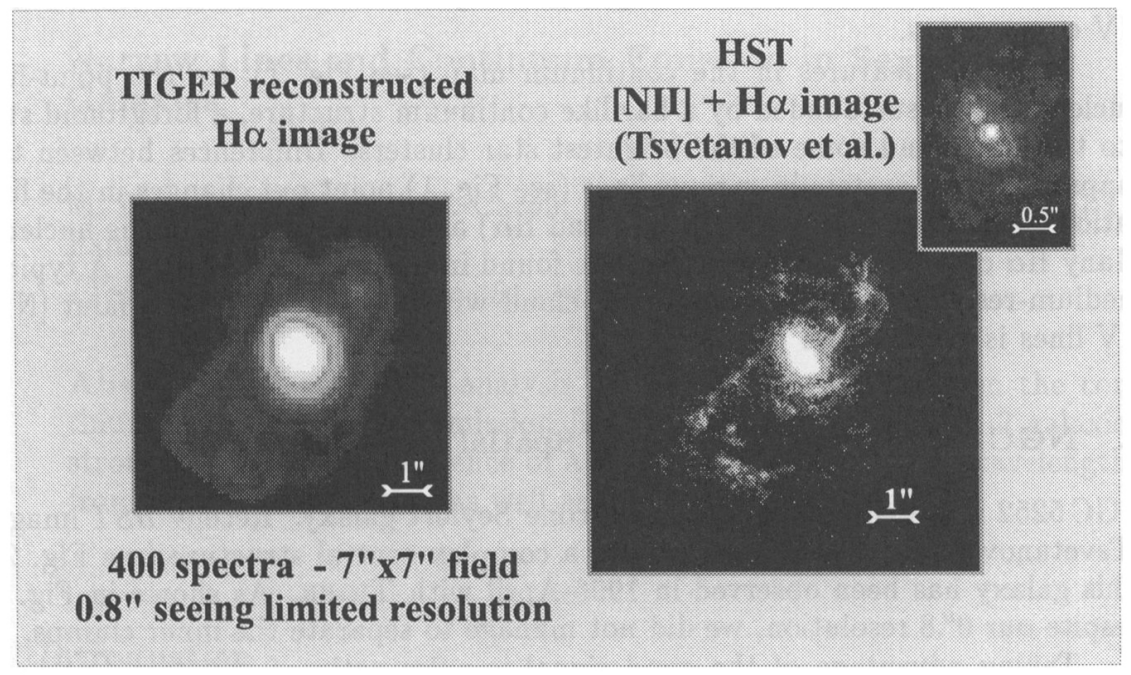

Figure 2. TIGER $\mathrm{H} \alpha$ map and $H S T$ narrow-band image.
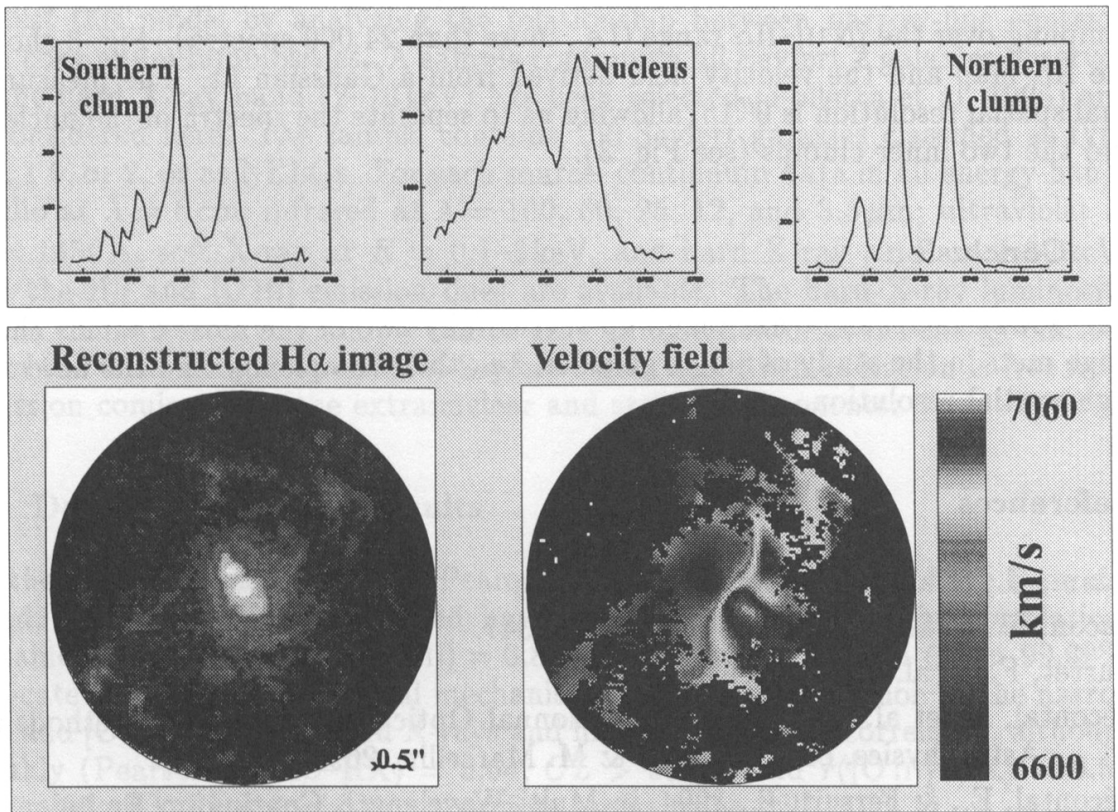

Figure 3. Top: Spectra (averaged over $0^{\prime \prime} .15 \times 0^{\prime \prime} .15$ areas) of the nucleus and of the two central clumps. Bottom: $\mathrm{H} \alpha$ image and velocity field derived from a Gaussian fit of the TIGER deconvolved 3-D data cube. 
CFHT. These data allow us to map separately the continuum and the LV and HV components.

The main features in the continuum map (see Fig. 1) are the point-like nucleus that is surrounded by a bar-like continuum structure, a foreground star (to the east), and some of the brightest star clusters. Differences between the maps of the LV system in various lines (see Fig. 1) point out changes in the line ratios (specially between [N $\mathrm{NI}] \lambda 6583$ and $\mathrm{H} \alpha$ ) as we go westward of the nucleus. Many $\mathrm{H} \alpha$ clumps of the HV system are found in our field (see Fig. 1). A typical medium-resolution spectrum of a $\mathrm{HV}$ cloud with the $\mathrm{H} \alpha$, [S II], and faint [N II] HV lines is displayed in Fig. 1.

\section{NGC 5252: The Challenge of Spatial Resolution}

NGC 5252 is a well-known ionization-cone Seyfert galaxy. Recent $H S T$ images (Tsvetanov et al. 1996) have revealed a complex central structure (see Fig. 2). This galaxy has been observed in 1996 April with TIGER. As shown in Fig. 2, despite our $0^{\prime \prime} .8$ resolution, we did not manage to separate the inner clumps.

Taking advantage of the good signal-to-noise ratio of the data, we have deconvolved the 3-D data cube. The HST image has been used to compute the spatial PSF. Then each velocity slice of the data cube has been deconvolved separately using a Lucy-Richardson-type method.

The NGC 5252 deconvolved data cube covers a $7^{\prime \prime} \times 7^{\prime \prime}$ area with $0^{\prime \prime} .045$ sampling over the $[\mathrm{N}$ II $]-\mathrm{H} \alpha$ range (i.e., more than 24,000 spectra). Fig. 3 shows the $\mathrm{H} \alpha$ map and the velocity field derived from a Gaussian fit. The measured final spatial resolution is $0^{\prime \prime} .15$, allowing us to separate the spectra of the nucleus and the two inner clumps (see Fig. 3).

\section{Conclusion}

The TIGER instrument has taken up successfully two of the most difficult challenge mets in the study of active galaxies, i.e., their complexity and the need for high spatial resolution.

\section{References}

Adam, G., et al. 1996, submitted to A\&A.

Bacon, R., et al. 1995, A\&A Supp., 113, 347.

Durret, F., et al. 1994, A\&A, 291, 392.

Pécontal, E., et al. 1995, in Tridimensionnal Optical Spectroscopic Methods in Astrophysics, ed. G. Comte \& M. Marcellin, 269.

Pécontal, E., \& Ferruit, P., 1994, in Multi-Wavelength Continuum Emission of AGN, ed. T. J.-L. Courvoisier \& A. Blecha, 445.

Petitjean, P., et al. 1996, Nature, 380, 411.

Rocca-Volmerange, B., et al. 1994, A\&A, 292, 20.

Tsvetanov, Z.I., et al. 1996, ApJ, 458, 172. 